Abstract 184 Table 1 Comparison of number of PED presentation and hospital admissions of children with acute wheezing between January-March and April-June 2018 to 2020

$201820192020 \quad \begin{aligned} & \text { Difference in } \\ & \text { percentage* }\end{aligned}$

Total number of PED

presentations with acute

wheezing, $\mathbf{n}$

- January-March

April-June

$\begin{array}{llll}507 & 671 & 557 & -5 \%\end{array}$

Total number of hospital

admissions, $\mathbf{n}(\%)$

January-March

April-June

$303(60 \%) \quad 430(64 \%) \quad 347(62 \%) \quad-5 \%$

$247(58 \%) \quad 436(63 \%) \quad 19(23 \%) \quad-95 \%$

PED presentations of children

under $5, \mathrm{n}$

- April-June

$\begin{array}{llll}360 & 507 & 378 & -13 \%\end{array}$

Hospital admissions of

children under 5, n (\%)

- January-March

- April-June

$241(67 \%) \quad 345(68 \%) \quad 256(68 \%) \quad-13 \%$

$173(62 \%) \quad 336(65 \%) \quad 10(25 \%) \quad-96 \%$

PED presentations of children

over $5, \mathrm{n}$

- April-June

147

164

148

183

179

$+15 \%$

Hospital admissions of

children over $5, \mathrm{n}(\%)$

- January-March

$62(42 \%) \quad 85(52 \%) \quad 91(51 \%) \quad+24 \%$

- April-June

$74(50 \%) \quad 100(55 \%) \quad 9(20 \%) \quad-90 \%$

*Difference in percentage is calculated by the difference between mean of 2018 and 2019 compared to 2020.

indefinitely sustainable, they demonstrate the potential health benefits of improved adherence to hand hygiene, pharmaceutical therapy, and sensible social distancing.

\section{IMPROVING THE ACUTE MANAGEMENT OF CHILDREN \& YOUNG PEOPLE (CYP) WITH EATING DISORDERS (ED) WITHIN A PAEDIATRIC TEAM}

Prisila Ahmed, Teizeem Dhanji, Victoria Chapman, Su Laurent. UK

\subsection{6/bmipo-2021-RCPCH.99}

Background Eating disorders are the third most chronic conditions in adolescents after asthma and obesity. Anorexia nervosa has the highest mortality of all psychiatric disorders, with cause of death being from the consequences of starvation or suicide. The condition has significant repercussions for the whole family unit. Since the start of the pandemic, there has been a dramatic increase in the number of CYP presenting with an ED requiring admission.

The Eating Disorders service for North Central London involves young people being admitted to the Paediatric ward at Royal Free Hospital (RFH) for management of re-feeding syndrome, medical instability and co-morbid mental health conditions.
CYP with Eating Disorders are at risk of fragmented care, particularly due to limited understanding of the disorder and unclear ownership over clinical management when admitted onto a paediatric ward. This requires change, and we have taken significant steps to harmonise the care of CYP within RFH.

\section{Objectives}

1. To raise awareness and understanding amongst staff managing CYP presenting with Eating Disorders to the RFH Paediatric ward.

2. To work as a multidisciplinary team of doctors, nurses and psychiatrists to improve the management of CYP with ED and consequently improve their quality of care.

Methods A new pathway has been implemented for management of CYP with Eating Disorders presenting to A\&E, including guidelines for admission for re-feeding. A new proforma has been introduced, along with a nurse's care plan, daily ward round sheet and re-feeding plan. Additionally, there are now two Consultant Paediatrician- led clinics per week for CYP who need an urgent or nonurgent medical assessment.Moreover, there is a teaching programme for doctors and nurses, including nurses having a four- week attachment with the ED team; providing comprehensive training on managing CYP admitted with Eating Disorders.

A questionnaire was sent to doctors and nurses prior to, and 1 year post- implementing the changes, to assess how their understanding has changed.

Results Following implementation of the pathway, proforma and teaching sessions, there was a dramatic improvement in confidence levels of all staff in managing children with ED. Initially, 38\% of people felt 'not confident' managing children with ED which dropped to $0 \%$ after the measures. There was an increase from $57 \%$ to $67 \%$ feeling 'fairly confident' and increase from $5 \%$ to $33 \%$ of staff feeling 'very confident'. Additionally, all staff knew where to find the guidelines for refeeding syndrome after the teaching sessions and all staff knew some of the Junior MARSIPAN criteria, compared to only $29 \%$ prior to these measures. There was a $20 \%$ increase in staff who were now aware that overall responsibility of care of a CYP admitted to the ward lies with the paediatric team with input from CAMHS.

Conclusions The strategies we have implemented show a definite improvement in the understanding of CYP with Eating Disorders and an awareness of the management and support they require from the multi-disciplinary team. Collaborative working between Paediatricians, CAMHS and Nursing staff is fundamental in the long- term management of these children and young people, achieving quality of care and positive outcomes.

\section{ASSOCIATION OF AUTOIMMUNE DISEASE AND INTUSSUSCEPTION WITH KBG SYNDROME - A RARE CASE REPORT}

Apurva Hegde, Darshanika Thennakoon Gamage, Kosmas Kailidis. UK

\subsection{6/bmjpo-2021-RCPCH.100}

Background KBG syndrome is a rare autosomal dominant genetic condition(Morel Swols et al., 2017) characterized mainly by skeletal abnormalities, distinctive facial features, and intellectual disability(Anon, n.d.) with a total of 150 reported 
cases(Anon, n.d.) in literature. Affected members in family show variable penetration(Kumar et al., 2009; Herrmann et al., 1975).

Coeliac disease (gluten sensitive enteropathy) is an autoimmune condition that primarily affects the small intestine and occurs in people at any age who become sensitive to a protein called gluten in their diet(Burden et al., 2014, Anon, n.d.).

This is the first reported case of KBG syndrome complicated with recurrent urinary tract infections(UTI), vesicoureteral reflux(VUR), anal stricture, recurrent intussusception and Coeliac disease.

Objectives To show the association of autoimmune disease and intussusception with KBG syndrome.

Methods A 16-year-old girl, diagnosed with KBG syndrome presented with recurrent abdominal pain, loose watery stools and loss of appetite for 1 year.

She presented to the hospital with recurrent UTI due to VUR from the age of 3 weeks up to date and had suffered from difficulties in passing stools from birth. However, an anal stricture was diagnosed and an ileostomy was performed at the age of 5 years. The genetic diagnosis of KBG syndrome was confirmed at the age of 10 years. Percutaneous endoscopic gastrostomy (PEG) was performed to manage feeding difficulties at the age of 12 years and she had a history of several spontaneously resolving intussusception episodes since last year. Intellectual disability, behavioral problems and developmental delay specially speech have been part of this problem(Morel Swols et al., 2017).

Her clinical examination in the recent admission was unremarkable except for pallor and right sided abdominal tenderness. The baseline investigations showed iron deficiency anemia and other causes for failure to thrive were ruled out. In addition to radiological diagnosis(by MRI) of intussusception, the second line investigations for abdominal pain and loose stools showed positive for anti-TTG antibody and which was later confirmed by genetic tests (HLA-DQ2 + HLA-DQ8) for coeliac disease.

Results Following the gluten free diet, her abdominal symptoms has shown improvement.

Conclusions KBG syndrome was diagnosed at a later stage in this patient due to the rareness of the condition. This patient has been suffering from the most of the complications of the disease out of which developmental delay and personality changes, led to delayed diagnosis of the abdominal pathology. The further evaluations with endoscopy procedures has been planned. Associated risk of autoimmune diseases and intussusception with KBG syndrome is yet to be identified.

\section{COMPARISON BETWEEN INFANTS AND OLDER CHILDREN WITH COVID-19 INFECTION}

Kah Wee Teo, Khuen Foong Ng, Srini Bandi. UK

\subsection{6/bmipo-2021-RCPCH.101}

Background Children are less likely to be severely affected or symptomatic from Severe Acute Respiratory Syndrome Coronavirus 2 (SARS-CoV-2) compared to adults. Currently, there is limited evidence on direct comparison of the presentation, clinical features, and managements between infants and older children.
Objectives To understand the difference between the impact of COVID-19 on infants (less than 1 year old) and older children (1 year to 16 years old)

Methods A single-centre retrospective cohort study evaluating children with confirmed COVID-19 who were admitted to University Hospitals of Leicester (UHL), United Kingdom between March to June 2020.

Results Between March to June 2020, a total of 17 children had been hospitalised with subsequent positive SARS-CoV-2 RNA PCR, of which 59\% were infant $(n=10)$. Older children had significantly higher CRP compared to infants. All older children (7/7) had elevated CRP ranging between 8 $\mathrm{mg} / \mathrm{L}$ to $403 \mathrm{mg} / \mathrm{L}$. $71.4 \%$ (5/7) of them had CRP more than $100 \mathrm{mg} / \mathrm{L}$. Among the infants, 50\% (5/10) had normal CRP (CRP $<5 \mathrm{mg} / \mathrm{L}$ ) and 30\% (3/10) had no blood tests taken during admission as they were clinically well. Higher proportion of older children with COVID-19 required admission into paediatric intensive care unit (PICU) or high dependency unit (HDU) and had significantly longer hospital stay compared to infant.

Abstract 187 Table 1 Differences in clinical features, investigation results, management and outcomes between infants and older children with COVID-19.

\begin{tabular}{|c|c|c|c|c|}
\hline & $\begin{array}{l}\text { Infant } \\
\text { (<12 } \\
\text { months) } \\
\mathrm{N}=10\end{array}$ & $\begin{array}{l}\text { Older children } \\
\text { (1-16 years } \\
\text { old) } \\
\mathrm{N}=7\end{array}$ & $p$-value & Statistical test \\
\hline Fever, $\mathbf{n}(\%)$ & $6(67)$ & $3(33)$ & 0.637 & Fisher's Exact \\
\hline $\begin{array}{l}\text { Respiratory symptoms, } \mathrm{n} \\
\text { (\%) }\end{array}$ & $5(63)$ & $3(38)$ & 1 & Fisher's Exact \\
\hline $\begin{array}{l}\text { Gastrointestinal symptoms, } \\
\text { n (\%) }\end{array}$ & $1(25)$ & $3(75)$ & 0.25 & Fisher's Exact \\
\hline Co-morbidities, n (\%) & $1(33)$ & $2(67)$ & 0.537 & Fisher's Exact \\
\hline CRP mg/L, median (range) & $5(5-25)$ & $135(8-403)$ & 0.003 & Mann-Whitney \\
\hline Abnormal CXR, n (\%) & $2(40)$ & $3(60)$ & 1 & Fisher's Exact \\
\hline Antibiotic usage, n (\%) & $5(42)$ & $7(58)$ & 0.044 & Fisher's Exact \\
\hline Oxygen requirement, n (\%) & $1(25)$ & $3(75)$ & 0.25 & Fisher's Exact \\
\hline PICU/HDU admission, n (\%) & $1(20)$ & $4(80)$ & 0.101 & Fisher's Exact \\
\hline $\begin{array}{l}\text { LOS in days, median } \\
\text { (range) }\end{array}$ & $3(1-7)$ & $12(4-22)$ & 0.002 & Mann-Whitney \\
\hline
\end{tabular}

CRP - C-Reactive Protein; CXR - Chest X Ray; HDU - High Dependency Unit; LOS - Length of Stay; PICU - Paediatric Intensive Care Unit

Conclusions Between March and June 2020, hospitalised older children in UHL appeared to be more adversely affected by COVID-19 compared to infants. This may correlate with the emergence of Paediatric Inflammatory Multisystem Syndrome Temporally associated with SARS-CoV-2 (PIMS-TS) and increased incidence of Kawasaki-like disease. Previous findings of infants having more severe disease from COVID-19 by Dong et al might be misleading as it predated the emergence of PIMS-TS and Kawasaki-like disease. The intention of this report is to alert the paediatric community that older children are likely to have a more severe disease when admitted with COVID-19. As this disease is a relatively new entity with evolving clinical picture, clinicians should be open-minded and remain vigilant. 